\title{
Is daily inhaled steroid use necessary in the treatment of mild persistent asthma?
}

Boushey HA, Sorkness CA, King TS, Sullivan SD, Fahy JV, Lazarus $\mathrm{SC}$, et al. Daily versus as-needed corticosteroids for mild persistent asthma. N Engl J Med 2005;352:1519-28.

Background: For years, daily anti-inflammatory therapy has been recommended for all cases of mild persistent asthma, yet asthma patients commonly fail to adhere to this daily regimen. Whether intermittent use can produce acceptable outcomes in this population is unknown.

Design: This multicentre, randomized, double-blind trial enrolled 225 adults with mild persistent asthma (defined as a forced expiratory volume in the first second $\left[\mathrm{FEV}_{1}\right]$ at least $70 \%$ of the predicted value, plus 1 or more of: as-needed $\beta$-agonist use more than twice a week but not daily; nocturnal awakening with asthma more than twice a month but not weekly; and variability in diurnal peak expiratory flow [PEF] of $20 \%-30 \%)$. Patients received either budesonide, 200 $\mu \mathrm{g}$ inhaled twice daily; zafirlukast (a leukotriene inhibitor), $20 \mathrm{mg}$ orally twice daily; or placebo. All of the patients were told to use a short-acting $\beta$-agonist as needed and received a written symptom-based action plan providing explicit instructions to start open-label inhaled budesonide therapy for 10 days or oral prednisone therapy for 5 days if their asthma symptoms worsened beyond specified clinical thresholds. Patients were followed for 1 year. The primary outcome was change in morning PEF. Secondary outcomes included change in $\mathrm{FEV}_{1}$, frequency of asthma exacerbations, degree of asthma control, number of symptom-free days and asthma-related quality of life.

Results: The morning PEF improved by similar amounts in the 3 study groups (budesonide 8.3\%, zafirlukast, $7.9 \%$ and placebo $7.1 \%, p=0.90)$. Compared with daily zafirlukast therapy or intermittent therapy (placebo group), daily budesonide therapy resulted in 26 additional symptom-free days per year $(95 \%$ confidence interval $1.8-48.5, p=0.03$ ) and was associated with better asthma control, less airway reactivity and greater decreases in inflammatory markers. No significant differences were observed between the groups in $\mathrm{FEV}_{1}$ after bronchodilator use, asthma exacerbations or asthma-related quality of life.

Commentary: The study's objective was not to redefine the best therapy for mild persistent asthma but, rather, to determine whether a treatment strategy imitating typical patient use produced acceptable outcomes. The reluctance of many asthma patients to adhere to inhaled steroid therapy, arising from concerns such as cost, adverse effects and inconvenience, is a reality of practice. However, many other patients would consider the additional symptom-free days and improved asthma control to be important enough benefits to make inhaled steroid therapy worthwhile. Although it is reassuring that intermittent treatment may not have serious consequences (at least in the short term), satisfactory outcomes must not be confused with optimal ones.

Since previous randomized trials have shown substantial benefits with inhaled steroid use in mild persistent asthma, ${ }^{1,2}$ including a reduction in exacerbations, why did the present study find more modest effects? Most likely patients in this study had less severe asthma than patients had in the previous studies. In fact, the investigators selected their cohort through rigorous screening. Even so, $15 \%$ of the enrolled patients were later ex- cluded after being found to have more severe asthma during the run-in period. It thus seems unlikely that sufficiently accurate characterization of asthma severity will be achieved in routine practice, which raises concerns about the generalizability of the study's findings. Also, all of the patients received a written selfmanagement plan, another important difference from previous studies. This probably enhanced the ability of the patients to cope with changes in symptoms and may explain in part why all of the groups had improved outcomes regardless of treatment, which consequently attenuates any effects of the study medications.

Practice implications: For patients with mild persistent asthma who do not wish to take inhaled steroid therapy daily, an as-needed treatment strategy may be acceptable, provided it is accompanied by education and an action plan with clear instructions about how and when to increase medication use. Nevertheless, daily inhaled steroid treatment remains the preferred evidence-based therapy because of its clinical benefits demonstrated in this and prior studies. ${ }^{1,2}$

\section{Matthew B. Stanbrook \\ Division of Respirology \\ Department of Medicine \\ University Health Network \\ University of Toronto \\ Toronto, Ont.}

Competing interests: Matthew Stanbrook received travel assistance to attend a meeting sponsored by AstraZeneca.

\section{References}

1. Pauwels RA, Pedersen S, Busse WW, Tan WC, Chen YZ, Ohlsson SV, et al; START Investigators Group. Early intervention with budesonide in mild persistent asthma: a randomized, double-blind trial. Lancet 2003;361:1071-6.

2. O'Byrne PM, Barnes PJ, RodriguezRoisin R, Runnerstrom E, Sandstrom $\mathrm{T}$, Svensson K, et al. Low dose inhaled budesonide and formoterol in mild persistent asthma: the OPTIMA randomized trial. Am 7 Respir Crit Care Med 2001;164:1392-7. 